\section{Acute psychosis due to brucellosis: a report of two cases in a rural Iran}

\section{Bidaki R, Yassini SM, Maymand MT,* Mashayekhi M, Yassini S}

Department of Psychiatry, Rafsanjan University of Medical Sciences, Rafsanjan, Iran

*Corresponded to: Dr. Mohammad Talebi Maymand Department of Psychiatry, Rafsanjan University of Medical Sciences, Rafsanjan, Iran; email: talebi22@gmail.com, Tel. No.: (+98)-3915230081, Fax: (+98)-3915230086

\begin{abstract}
INTRODUCTION: Brucellosis is a zoonotic disease that causes multi systemic involvement. Neurobrucellosis occurs in less than $5 \%$ of patients. Psychosis as a presentation of neurobrucellosis is a rare condition.
\end{abstract}

CASE REPORT: We report a woman that was referred because of psychotic depression following brucellosis. Also we present a 66-yearold man that was admitted because of acute psychotic symptoms. He had behavioral disorders, visual and auditory hallucination, restlessness, impulsivity, incoherency and episodic crying. Neurobrucellosis was confirmed.

CONCLUSION: In patients with atypical psychosis in endemic areas, physicians should consider the portability of brucellosis.

KEY WORDS: Neurobrucellosis, Psychiatry, Infectious disease

Article submitted 29 January. Reviewed 28 February. Author correction 13 March. Final version accepted 28 March 2013. 


\section{INTRODUCTION}

Brucellosis is a zoonotic disease that might causes multi organ involvement. Central nervous system involvement is rare but may causes serious clinical manifestations. Neurobrucellosis occurs in less than $5 \%$ of patients. ${ }^{1}$ Transmission of brucellosis to humans occurs through the consumption of infected, unpasteurized animal milk and its products, through direct contact with infected animal parts, through ruptures of skin and mucousal membranes and inhalation of infected aerosolized particles. $^{2}$

The involvement of the central nervous system (CNS) has a wide range of presentations. Subacute and chronic meningoencephalitis are described as the most common neurologic presentations. ${ }^{3}$ In epidemic regions for brucellosis, if uncommon, unexplained and ambiguous manifestations are seen, a brucellar psychosis should be considered. ${ }^{4}$ Psychosis as a manifestation of neurobrucellosis is a rare status. Here we report two brucellosis cases with psychotic symptoms.

\section{CASE PRESENTATION 1}

A 25 years-old pharmacist married women developed generalized headache, nausea, low back pain and restlessness which continued for two months that her headache became more severe and developed three episodes of brief unconsciousness without tonic or colonic movement. Brain Magnetic Resonance Imaging (MRI), Complete Blood Count (CBC), serum calcium and phosphorus, Cerebro Spinal Fluid (CSF) and Fasting Blood Sugar (FBS) were normal. After a few days she developed auditory hallucination, insomnia, dysphoria, decreased her interest in activities (job performance and interpersonal relationships). Tablet haloperidol $(10 \mathrm{mg})$ and imipramine $(25 \mathrm{mg})$ were initiated with impression of psychotic depression but there was no response till one week. Electro-Compulsive Therapy (ECT) was tried but after the third ECT she became worse and developed fever and redness and swelling over the left parotid gland. Medication and ECT were discontinued and following consultation with an infectious specialist and based on antibody tests (IgM antibodies positive for Brucella spp.), brucellosis and encephalitis approved as final diagnosis according to the blood and CSF tests. Other tests were negative which excluded other possible infectious causes. During the work up process the level of consciousness of the patient decreased gradually and finally developed coma. Rosetresia and piperacillin were started and after
23 days she became afebrile, conscious, and alert and was discharged with doxycycline, rifampine and gentamycin. She was symptom free for 10 years and again developed depressed mood, right flank pain, chilly sensation and sweating. She was again treated with rifampin, doxycycline and gentamicine. This episode reoccurred in 4 consequent years improved with same medication but no problem since 1998.

\section{CASE PRESENTATION 2}

A 66-year-old man, married, farmer, from Rafsanjan city (South of Iran) was admitted to our psychiatric ward because of acute psychotic symptoms. In past history, he had no psychiatric disorders. There was a history of bipolar disorder in his son. Also there was multiple histories of brucellosis in his family members. One week before his psychiatric presentation, he developed headache, ataxia, malaise, fatigue, irrelevant speech and joint manifestations. Brain MRI was normal, neutrohil was high $(12,900 \mathrm{cmm})$, CSF was norma, IgM test for brucellosis was positive. The diagnosis of neurobrucellosis was proposed and was empirically treated with doxycycline, and co-trimoxazole. Then he was transferred to our psychiatric ward for treatment of psychotic symptoms. On arrival to our psychiatric ward, his physical examination was normal.

He had behavioral abnormalities such as restlessness, impulsivity, and episodic crying. His speech was incoherent. It seemed that he was driving in a car and he believed that nurses injected the worms in his serum. His cognition was impaired and had poor insight. Three day after the treatment with risperidone (1mg HS), haloperidole $(2.5 \mathrm{mg}$ BID) and sodium-valproate (200mg OD), his impulsive behavior, restlessness, an psychotic symptoms were decrease and finally were resolved.

\section{DISCUSSION}

Neurologic involvement is rare that happens in 2 $5 \%$ of brucellosis. Neurologic signs can present during the active or delayed period of disease. Diagnosis are suggested on the basis of history of the exposure, cerebrospinal fluid analysis and serology (IgM antibody). ${ }^{5}$ Depression is the most common psychiatric disorder in brucellosis. In acute brucellosis similar to the other febrile illnesses, delirium may occur. ${ }^{6}$ In every two cases, psychosis is developed in acute phase of illness. We ruled out delirious state in this stage because of intact cognition. Hallucinations and delusions might be the primary presentation of brucellosis. ${ }^{7}$ In every two 
cases, misperception and disturbance in content of thought are developed in acute phase of illness.

In Shehata study, depression has been reported as the most common presentation of neurobrucellosis. ${ }^{8}$ In our study, a depressed mood was detected too. The psychiatric presentations that are reported include: depressed mood, amnesia, agitation, nightmares, personality traits, euphoria, misperception, poverty of content of thought, hallucination, delirium, dysarthria, psychotic symptoms. ${ }^{9,10}$ We saw all presentations in our cases. Therefore psychiatric manifestations are predictable and common in Neurobrucellosis.

These two cases suggest that brucellosis could present in extended clinical forms. Therefore physicians should think of brucellosis in patients who experience unexplained signs and symptoms that include neurological and psychiatric problems when there is a history of a probable exposure with brucella.

\section{CONCLUSION}

We concluded that psychotic symptoms probable follow after acute brucellosis. Response to antipsychotic therapy is favorable in this condition. In endemic areas, brucellosis should be considered in patients with psychosis.

CNFLICT OF INTEREST: None to declare

FINANCIAL INTEREST: None to declare

\section{REFERENCES}

1. Baykal T, Baygutalp F, Senel K, et al. Spastic paraparesis and sensorineural hearing loss in a patient with neurobrucellosis. J Back Musculoskelet Rehabil 2012;25:157-159.

2. Haque N, Bari MS, Hossain MA, et al. An overview of Brucellosis. Mymensingh Med J 2011;20:742-747.

3. Türel O, Sanli K, Hatipoğlu N, Aydoğmuş C, Hatipoğlu $\mathrm{H}$, Siraneci R. Acute meningoencephalitis due to Brucella: case report and review of neurobrucellosis in children. Turk J Pediatr. 2010;52:426-429.

4. Sheybani F, Sarvghad MR, Bojdi A, Naderi HR. Brucellar psychosis. Arch Iran Med 2012; 15:723-725.

5. Bellissima P, Turturici MA. Neurobrucellosis: clinical and therapeutic features. Infez Med 1998;6:25-30.

6. Izadi S. Neurobrucellosis. Shiraz E Med J 2001;1:1-7.

7. Amato E, Anasagasti I, Poza JJ, Yerobi JA. Acute psychosis as a manifestation of brucellosis. Enferm Infect
Microbiol Clin 1989;7:231-232.

8. Shehata GA, Abdel-Baky L, Rashed H, Elamin H. Neuropsychiatric evaluation of patients with brucellosis. J Neurovirol 2010;16:48-55.

9. Drevetes DA, Leenen P, Greenfield RA. Invasion of the central nervous system by intracellular bacteria. Clin Microbiol Rev 2004;17:323-347.

10. Sahin OG, Pelit A, Turunc T, Akova YA. Ophthalmoparesis, papillitis and premacular hemorrhage in a case with endocarditis: a rare presentation of brucellosis. Indian J Ophthalmol 2010;58:164-166.

\section{Citing this article}

Bidaki R, Yassini SM, Maymand MT, Mashayekhi M, Yassini S. Acute psychosis due to brucellosis in a rural Iran: a report of two cases. J Inter Infect Microbial 2013;2(1):29-31. 